\title{
Editing misleading information from memory: Evidence for the coexistence of original and postevent information
}

\author{
ROBERT E. CHRISTIAANSEN and KATHLEEN OCHALEK \\ Lawrence University, Appleton, Wisconsin
}

\begin{abstract}
When misleading postevent information biases one's memory for an event, what is the fate of the original, accurate information? One possibility is that the new information and the original information coexist in memory, but that the former is simply more accessible. A second hypothesis suggests that the new information replaces the old, and memory is irreversibly altered. Using various retrieval techniques, Loftus and her associates (Greene, Flynn, \& Loftus, 1982; Loftus, 1979a, 1979b) have failed repeatedly in attempts to recover original memories after postevent biasing, a finding that supports the alteration hypothesis. In the present study, postevent biasing was demonstrated in two experiments. In each experiment, some subjects were given a warning that the postevent information had included a few inaccurate details. These subjects were able to edit out the inaccurate details and to recover the original facts when the warning came as much as $\mathbf{4 5} \mathrm{min}$ after they had read the misleading information, a result that argues for the coexistence of memories. Successful recovery of the original memories was apparently due to the clarity of the warning and to an improved technique for assessing the retrieval of original memories.
\end{abstract}

In recent years, numerous studies have demonstrated that misleading postevent information introduced in a narrative or as a presupposition of a question can alter a person's recollection of an event (e.g., Christiaansen, Sweeney, \& Ochalek, 1983; Dodd \& Bradshaw, 1980; Loftus, 1975; Loftus \& Greene, 1980; Loftus, Miller, \& Burns, 1978; Loftus \& Palmer, 1974; Powers, Andriks, \& Loftus, 1979). Although it is clear that such postevent information has an effect, questions remain about why the false postevent information is remembered instead of the original, when it is demonstrated (or at least reasonably certain) that the original information was indeed encoded in memory. Perhaps the original information still exists in memory, but is simply less accessible than the more recent biasing information. Loftus (1979a) referred to such an explanation as the coexistence hypothesis, since the original and postevent information coexist in memory. Alternatively, one's memory could actually be changed and updated, and the original representation destroyed, each

This research is based on an honors thesis by the second author under the supervision of the first. We wish to thank Audrey Skaife and the University of Wisconsin-Oshkosh for providing subjects for Experiment 2, and Elizabeth F. Loftus for providing copies of her event slides used in that same experiment and for additional materials used in constructing the narratives and tests. We also wish to thank D. James Dooling, William D. Francis, Elizabeth F. Loftus, and Illene C. Noppe for commenting on an earlier draft of this paper. Requests for reprints should be sent to the first author at the Psychology Department, Lawrence University, Appleton, Wisconsin 54912. time new information about an event is encountered. This view Loftus has labeled the alteration hypothesis.

In an effort to test these two hypotheses, Loftus and her associates have conducted several experiments to try to recover original information from memory after postevent biasing. This should be possible according to the coexistence hypothesis, but not according to the alteration hypothesis. Various techniques have been employed to attempt such recovery, and, to date, none has been successful (see Loftus, 1979a). Perhaps the most direct approach has been the use of warnings about the misleading postevent information (Greene, Flynn, \& Loftus, 1982; Loftus, 1979b). In these studies, after having witnessed an event and read a misleading narrative about it, subjects were given warnings suggesting that there might have been incorrect information in what they had read. If subjects could use such warnings and respond with the correct, original information instead of being misled (as unwarned control subjects typically are), then one would have evidence that the original and the misleading information coexist in memory. Detecting the discrepant details and reporting the correct information requires comparing the two coexisting memory representations, one from the event and the other from the narrative. The warnings used have been either implicit, in the form of a blatant (and noticeable) contradictory statement in the narrative (Loftus, 1979b), or explicit, simply stating that some of the information may have been inaccurate (Greene et al., 1982). In both cases, the warnings were found to be effective only if they preceded the subtle misleading 
information, not when they followed the reading of that information. Thus, subjects could apparently detect inaccurate statements in the narrative if they were warned, but could not edit out these inaccuracies once encoded in memory.

Despite these and other failures (Loftus, 1979a) to find evidence for coexistence, we felt there were several reasons to question the rejection of this hypothesis and the acceptance of the alteration hypothesis. Our first concern was a problem clearly acknowledged by Loftus and her associates (Loftus, 1979a; Loftus \& Loftus, 1980). Although attempts to recover original memories potentially can provide evidence for coexistence if the original information is successfully recovered, failure to recover original memories is only suggestive evidence in favor of alteration. One can always argue that the original memory still exists but that the appropriate retrieval method was not used. This logical difficulty alone might be justification enough for a few more attempts at demonstrating coexistence. But our concern went beyond a feeling that "one more try" was needed. Two aspects of the previously described studies might well have affected the chances of successfully demonstrating coexistence of memories.

First of all, an even more explicit warning than that used by Greene et al. (1982) may be necessary to ensure that subjects are motivated to search memory for inconsistent pieces of information. Greene et al. told subjects that some of the information in the narrative they read "may have been inaccurate" because the police cadet (who supposedly had written it) was inexperienced. With a stronger warning, memory search should be perceived as more likely to succeed in finding inconsistencies. In addition, not mentioning a cause for the errors should eliminate the possibility that subjects dismiss the "warning" as unimportant to their task and think of it as a mere notation of some accidents for which the experimenter is offering an explanation. One purpose, then, of the present study was to attempt to recover coexisting memories by using an unambiguous warning that asserted that errors did exist in the narrative.

In addition to the clarity of the warning, the present study included an assessment of whether the original event information was actually encoded in memory. Proof that the original information was indeed encoded would seem to be desirable for any test designed to determine if subjects can recover such memories; yet, in all but one of the studies testing the various recovery techniques, no initial accuracy test was given before the biasing information was introduced. ${ }^{1}$ Without such a test, one cannot identify which specific information was encoded by each subject, and therefore what is potentially recoverable. Performance by control groups can, of course, indicate the general level of accuracy when no postevent information is introduced, but reliance on such estimates may introduce an artifact that reduces the apparent effect of the recovery technique. When the tests include items that a given subject may never have encoded, as must surely happen in these studies, this can only mitigate the success of the various recovery techniques. In the present study, an initial accuracy test assessed memory for the specific facts that were later biased, and only those items for which a subject was initially accurate were included in our principal analyses. Therefore, only those memories that were potentially recoverable were examined, thus providing a fair test for the effectiveness of the warnings.

\section{EXPERIMENT 1}

In Experiment 1, there were four conditions. The subjects in the neutral condition served as a control group and did not receive misleading information, whereas the subjects in the biased condition did receive misleading postevent information. The subjects in the biased-immediately-informed and biased-delayedinformed conditions also received misleading postevent information. In the former condition, the subjects were warned, immediately after having read the narrative, that they had read some inaccurate information, and in the latter condition, they received this same warning approximately $45 \mathrm{~min}$ later, just prior to the final test.

\section{Method}

\section{Subjects}

Sixty-eight students from Lawrence University and one individual from the city of Appleton participated in the study. The data from only 61 subjects were used because 8 of the students indicated, on debriefing questions, that they knew something about the purpose of the experiment from the outset. This high number was probably due to the fact that the experiment was done on a small campus on which previous memorybiasing research had been conducted.

\section{Materials}

Slides. The event to be remembered was a series of 24 color slides depicting an ambiguous shoplifting event in a department store. The slide sequence opens with a young man walking down the aisle of the store. After purchasing a small item, the young man walks through the sporting goods department and looks at a compass. As the manager of the store watches, the young man turns and appears to reach into his pocket. Later, the young man walks toward an exit with the item he previously has paid for, but the manager is standing in the doorway. Upon seeing the manager, the young man walks to the back of the store and into a room full of stereo equipment, with the manager following him. A struggle ensues in which the manager grabs the young man, but the young man gets away and runs out of the store.

Accuracy tests. The initial accuracy test consisted of 31 items that addressed diverse details of the event. With the exception of two open-ended questions, about the height and weight of the suspect, all items were declarative sentences with a missing phrase or word and four alternatives-the fourth frequently being "none of the above." Of the 31 items, four were critical: Three-fourths of the subjects would later read biased information about these four details. The final accuracy test contained 27 items similar to those on the initial test. Four were the identical critical items that had appeared on the first test. The height and weight questions and two other extraneous items were the only other questions exactly the same on both accuracy tests.

Narrative. The narrative was a very detailed step-by-step account of the slide sequence. It was the same for all subjects, except for the references made to four critical details. The sub- 
jects in the neutral condition read a narrative in which there was no mention of these four critical details, whereas the subjects in the biased, biased-immediately-informed, and biased-delayedinformed conditions read a narrative in which these four details were described inaccurately. For example, the slide sequence showed the young man walking past a display of vacuum cleaners. The narrative for neutral subjects stated, "After passing a display on his left, he walked through the catalog department, and then passed a rack of ribbons and wrapping paper." The biased version of the narrative stated, "After passing a display' of garden tools on his left, he walked through the catalog department, and then passed a rack of ribbons and wrapping paper." Of the four critical details, two concerned the existence of an object and two concerned actions. For none of the critical details was the biased version true of any portion of the event (e.g., for the sample question, no garden tools were present in any slide). ${ }^{2}$

\section{Procedure}

On Day 1, the subjects were shown the series of slides, for 5 sec each. They were told: "The slide sequence you are about to see involves three main characters: a young man, the manager of the store, and a cashier. Please look at the slides carefully because later you will be asked to determine if any crime was committed." Immediately after having viewed the slides, the subjects completed the initial accuracy test. The subjects were told to write the letter of the one best answer in the space provided and to rate their confidence for each answer on the scale provided. The scale ranged from 1 to 5 , with 1 indicating low confidence and 5 indicating high confidence. After the subjects had completed the test, they were asked not to discuss the slides or test with anyone, and they were instructed to return 2 days later at the same time.

On Day 2, approximately $48 \mathrm{~h}$ later, the subjects received an envelope that contained all of the test materials for the remainder of the experiment. They were told that they would be taking a series of personality and memory tests. The subjects then were instructed to take the first test, which was ostensibly a measure of their memory abilities, out of the envelope. This test contained the narrative. On the first page of the test, the subjects read the following questions: (1) "Assume that you had been asked to write a description of the event at this time (approximately two days after seeing the slides). What percentage of the information contained in the attached description do you think you would have recalled?" (A set of choices followed from $0 \%$ to $100 \%$.) (2) "It is highly unlikely that any of you would recall $100 \%$ of the information in the description since you only saw each slide for five seconds. How long do you think you would have had to look at each slide in order to give a description as detailed as that on the following page?" The second page of the test contained the narrative. The subjects were told to read through the questions on the cover page first, then to turn to the second page and read the description once only, and then, without looking back at the description, to answer the questions on the first page. After all subjects in a session (usually 3-10 subjects) had completed the questions, they were told to turn to the third page of the test. It was on the third page that the subjects in the biased-immediately-informed condition read a statement informing them that " $a$ few of the details in the description are inaccurate-some of the details are correct and a few are incorrect. Take a minute to think about the description, BUT DO NOT GO BACK AND LOOK AT THE DESCRIP. TION AND DO NOT WRITE ANYTHING DOWN. Just think about the description for one minute." The subjects were given just $1 \mathrm{~min}$ for this task. The time between reading the narrative and being informed in this condition ranged from $30 \mathrm{sec}$ to about $4 \mathrm{~min}$, since it was necessary to wait for all subjects in a session to complete the first two questions. So that all subjects would be doing something at this point in the experiment, the subjects in all the other conditions had to complete an unrelated problem on the third page.

Next, the subjects completed a series of five personality and list-learning tests designed to keep them occupied for $45 \mathrm{~min}$. After all subjects had finished these tests, they were instructed to complete the final accuracy test. For the subjects in the neutral, biased, and biased-immediately-informed conditions, a cover page simply stated some standard directions for the test, but for the subjects in the biased-delayed-informed condition, in addition to these directions, the cover page included a statement informing them that " $a$ few of the details in the description of the slide sequence, which you read at the beginning of the hour, were inaccurate-some of the details are correct and a few are incorrect." For all subjects, the directions stated that the subjects should answer the test items on the basis of the slide sequence only.

\section{Results and Discussion}

For each subject, his or her accuracy and confidence score for each critical item on the final test was used to calculate a combination score, ranging in value from 1 to 10. A combination score of 10 was assigned to a correct response given with a confidence rating of 5 (high confidence), a combination score of 9 was given to a correct response with a confidence rating of 4 , and so forth. A combination score of 1 was assigned to an incorrect response given with a confidence rating of 5 , which indicated that the subject had high confidence in an incorrect response. For each subject, the mean of these combination scores for the four critical items was then computed. For the principal analysis, however, the data were restricted to include only those items on the final accuracy test that the subjects had answered correctly on the initial accuracy test. As a result, although each subject contributed one score, it could be based on one to four responses, depending upon how many of the four critical items that that subject initially had answered correctly. Any subject initially incorrect on all four critical items would have been dropped from this analysis, but no subject had to be excluded for this reason.

Inspection of the mean combination scores shown in Table 1 indicates that the misleading postevent information had the expected influence on the subjects' performance on the four critical items. The subjects in the biased condition were less accurate than the subjects in the neutral condition. A one-way analysis of variance, done on the combination scores, yielded a significant effect of condition $[F(3,60)=20.62, p<.001] .^{3}$ Post hoc comparisons were performed using NewmanKeuls tests at the .05 significance level. The comparisons confirmed that the biased condition was significantly less accurate than the neutral condition. Regarding the effects of the warning, the biased-immediately-informed and the biased-delayed-informed conditions were both significantly more accurate than the biased condition. Warning the subjects that they had read some inaccurate information in the narrative did allow them to overcome the biasing effect of the misleading narrative. The 
Table 1

Mean Critical Item Scores for Initially Accurate Items in Experiment 1

\begin{tabular}{lcccc}
\hline & \multicolumn{4}{c}{ Experimental Condition } \\
\cline { 2 - 5 } & $\begin{array}{c}\text { Neutral } \\
(\mathrm{N}=16)\end{array}$ & $\begin{array}{c}\text { Biased } \\
(\mathrm{N}=15)\end{array}$ & $\begin{array}{c}\text { Biased/Immediately } \\
\text { Informed } \\
(\mathrm{N}=12)\end{array}$ & $\begin{array}{c}\text { Biased/Delayed } \\
\text { Informed } \\
(\mathrm{N}=18)\end{array}$ \\
\hline Combination Score & 8.72 & 4.46 & 6.51 & 8.94 \\
Proportion Correct & .95 & .41 & .62 & .95 \\
Proportion Biased & .05 & .59 & .26 & .05 \\
\hline
\end{tabular}

Note-Combination scores were computed such that a 1 equals a high-confidence incorrect response and a 10 equals a high-confidence correct response.

narrative and original event information, therefore, must have coexisted, since the subjects could edit out the inaccurate information and recover the original memories at the time of the warning.

If warned subjects are able to edit the inaccurate information from their memories completely, then the subjects in the two informed conditions should have been no less accurate than the subjects in the neutral condition, in which the subjects were never exposed to biasing statements. In fact, the subjects in the biased. delayed-informed condition did not differ significantly from those in the neutral condition, but the biasedimmediately-informed subjects were significantly less accurate than the subjects in both the biased-delayedinformed and the neutral conditions. The finding that those subjects who had been warned just before the final test were more accurate than the subjects who received the warning immediately after having read the incorrect information was quite unexpected. In retrospect, we suggest that this outcome may have resulted from our failure to repeat the warning for the immediate group just prior to the final test. These subjects were warned immediately after having read the biased narrative, but at the time of the final accuracy test, they were merely given the standard instructions. Perhaps between the time they received the warning and the time of the final test, some of these subjects simply forgot about the warning. This possibility was explored in Experiment 2.

In addition to the combination scores, two proportion scores were also computed, again restricted to only those items initially correct. As seen in Table 1, the pattern of mean proportions correct corresponds to that for the mean combination scores, and the mean proportions of biased answers (i.e., those corresponding to the misleading narrative statements) are virtually a mirror image of the mean proportions correct, indicating that inaccurate responses were indeed largely due to the subjects' incorporating the misleading details into their memories. Finally, although the table does not include data on initial test performance, it should be noted that the groups did not differ on the critical items at that time (i.e., prior to having read the narrative). The mean proportions correct for the four conditions, as shown in Table 1 , were $.74, .75, .68$, and .65 , respectively $(F<1)$.
A possible interpretation of the present findings is that the subjects who were warned simply doubted the accuracy of all narrative information once they had been told that the narrative contained inaccuracies; that is, they did not differentiate between accurate and inaccurate narrative information but, instead, just rejected everything they recalled reading as part of the narrative. This would not, of course, imply coexistence. We have two forms of evidence against this "narrative-rejection" hypothesis. First, it should be noted that even if this strategy were employed, it would result mainly in fewer biased answers and have less impact on the number of accurate responses. Once the biased, narrative answer is rejected for a critical test item, there remain three other answers from which to select. Any substantial increase in the number of correct answers still requires memory for the original event. Examination of Table 1 shows that the lower proportions of biased answers in the two informed conditions, compared with those in the biased condition, were coupled with correspondingly higher proportions correct. This argues for true memory editing instead of merely nonselective rejection of narrative information.

The other evidence against the narrative-rejection hypothesis comes from data collected on noncritical test items. If subjects reject all narrative information when they are warned, then they should be less accurate than nonwarned subjects on questions about other facts described accurately in the narrative, since rejection of this narrative information must result in errors. To test this prediction, several such questions were included on the final test. Not all of these items were usable, however. We realized after having completed the experiment that only items for which subjects' initial accuracy had been assessed could provide appropriate data. Since the principal data of the experiment concern only initially accurate items, the narrative-rejection strategy, if it is to explain the effects of the warning, must apply to items of this type as well. In other words, subjects must be willing to reject answers that they had correct on the initial test, simply because they recall those facts as being stated in the narrative. There were a total of eight questions that occurred on both the initial and final tests and that asked about information accurately described in the narrative (recall that, except for the four 
critical details, the narrative did not differ across conditions, so these eight facts were described identically for all subjects). The questions were not precisely the same from the initial to the final test in six of the eight cases, but the differences were only minor wording changes. Using these eight questions, a combination score and proportion correct were computed for each subject by the same procedure that was used for the critical questions. The mean scores for each condition are shown in Table 2. As is clear from Table 2, and was confirmed by an analysis of variance, there were no differences among these groups. The warned subjects apparently did not adopt a strategy of rejecting all information that they recalled being in the narrative, or they would have done poorly on these accurately described facts.

The results of Experiment 1 support the coexistence hypothesis, which claims that both the information about the original event and the conflicting postevent information can coexist in memory. Our subjects, when given a clear warning that the narrative contained in accuracies, could retrieve the original information, compare it with the narrative information, and selectively edit out the inaccurate material. In an effort to provide the most powerful test for the effects of the warning, we restricted our analysis to only those items answered correctly on the initial test. As it turned out, an analysis using all four critical items for each subject yielded a similar pattern of results. However, by that analysis, the subjects in the informed conditions showed somewhat lower accuracy scores than those in the neutral condition, and the subjects in the biased-immediatelyinformed condition were not significantly more accurate than those in the biased condition. Thus, the inclusion of initially inaccurate items did reduce the apparent effect of the warning.

\section{EXPERIMENT 2}

Experiment 2 had two major objectives: first, to replicate the previous experiment using a completely different set of materials, and second, to try to clear up the ambiguity in Experiment 1 concerning the effects of an immediate warning. To eliminate the possibility of biased-immediately-informed subjects' forgetting the warning, in Experiment 2 a reminder was in- cluded for these subjects just prior to the final test.

Some additional changes were made in an effort to provide further evidence concerning the narrativerejection hypothesis. The most important of these changes involved the inclusion of a condition in which the subjects read accurate information in the narrative about the four critical details. These subjects were then warned, just before the final test, that the narrative contained some inaccurate information, making this an accurate-delayed-informed condition. If the warning results in a strategy of rejecting all narrative information as inaccurate, subjects in this new condition should not only reject noncritical narrative items (the test employed in Experiment 1), but should also reject the information about the four critical details. Since the information was accurate, this would lead to low accuracy on the final test. Thus, a new test of the narrative-rejection hypothesis was added, using the same four critical details as the test for bias effects.

\section{Method}

\section{Subjects}

The subjects for this study were 124 students enrolled in an introductory psychology class at the University of WisconsinOshkosh. The data from two subjects were eliminated due to their failure to follow directions. On the debriefing questionnaire, no subjects indicated prior knowledge of the manipulation.

\section{Materials}

A series of 24 color slides that depicted a wallet-snatching incident in a small town was shown to all subjects. These slides were the same ones used in the Loftus $(1979 \mathrm{~b})$ study of reactions to blatantly contradictory statements.

The initial and final tests consisted of 24 multiple-choice items each. Of these items, 4 were critical items, and the remaining 20 were of two types, narrative and nonnarrative. Narrative items tested for information that was included in the narrative description of the slide sequence. Nonnarrative items tested for information that was not mentioned in the narrative. Five narrative and five nonnarrative questions were exactly the same on both the initial and final accuracy tests. The remaining 5 items of each type appeared only on the initial test or only on the final test.

Except for the references made to four critical details, the narrative, a detailed step-by-step account of the slide sequence, was the same for all subjects. Statements about the four critical details varied with the condition. For example, in the slide sequence, two boys, one on a bicycle and one standing, are seen

Table 2

Mean Narrative Item Scores for Initially Accurate Items in Experiment 1

\begin{tabular}{ccccc}
\hline & \multicolumn{2}{c}{ Experimental Condition } \\
\cline { 2 - 6 } & $\begin{array}{c}\text { Neutral } \\
(\mathrm{N}=16)\end{array}$ & $\begin{array}{c}\text { Biased } \\
(\mathrm{N}=15)\end{array}$ & $\begin{array}{c}\text { Biased/Immediately } \\
\text { Informed } \\
(\mathrm{N}=12)\end{array}$ & $\begin{array}{c}\text { Biased/Delayed } \\
\text { Informed } \\
(\mathrm{N}=18)\end{array}$ \\
\hline Combination Score & 8.90 & 9.04 & 8.96 & .96 \\
Proportion Correct & .95 & .91 & .81 \\
\hline
\end{tabular}

Note-Combination scores were computed such that a l equals a high-confidence incorrect response and a 10 equals a high-confidence correct response. 
looking at a window display. The narrative for neutral subjects stated, "Behind her, two boys were looking at a window display." For the three biased conditions, it stated, "Behind her, two boys, one on a skateboard and one standing, were looking at a window display." The narrative for the accurate-delayedinformed condition stated, "Behind her, two boys, one on a bicycle and one standing, were looking at a window display." Of the four critical details, two concerned the existence of an object and two concerned the color of an object. The latter two details were the same ones used by Loftus (1979b), but the two object questions were different. For none of the critical details was the biased version true of any portion of the slide sequence.

\section{Procedure}

At the beginning of class, the subjects were shown the series of 24 color slides for $8 \mathrm{sec}$ each. The subjects were told, "The slide sequence you are about to see depicts a crime. Please view the slides very carefully because you will be asked questions about this event later." Immediately after having viewed the slides, the subjects completed the initial accuracy test. The subjects were then asked not to discuss the slides or the test for the next 2 days.

At the beginning of the next class, $48 \mathrm{~h}$ later, the subjects received an envelope that contained the two remaining tests. The subjects were instructed to take out the first test, which contained the narrative. They then completed the same task that had been used in Experiment 1, which served to introduce the narrative information. After all the subjects had completed this test, it was collected and the class lecture was begun.

Near the end of class, approximately $30 \mathrm{~min}$ later, the subjects completed the final accuracy test. The only difference in procedure from Experiment 1 was that the instructions reminded the biased-immediately-informed subjects that a few of the details in the description were inaccurate.

\section{Results and Discussion}

As in Experiment 1, a mean combination score was computed for the four critical items for each subject; only those items that had been answered correctly on the initial test were used on the final test. Nineteen subjects who had been initially incorrect on all four critical items were excluded from the analysis. The mean combination score for each condition, as well as proportions of correct and biased responses, can be seen in Table 3. A one-way analysis of variance performed on the combination scores revealed a significant difference among conditions $[F(4,98)=9.37, p<.001]$. Post hoc comparisons were performed on these means with the Newman-Keuls test at the .05 significance level. The subjects in the biased condition were found to be significantly less accurate than those in the neutral condition, indicating that biased subjects did incorporate the misleading information into their memories. The subjects in the biased-immediately-informed and the biased-delayed-informed conditions were both significantly more accurate than those in the biased condition, showing that the warning was once again effective. Furthermore, the subjects in the neutral condition and in the two informed conditions did not differ significantly in accuracy. Warning the subjects that they had read some inaccurate information yielded performance as accurate as the performance of subjects who never had encountered the misleading information in the first place. Thus, the editing ability of warned subjects was shown to be quite good. In Experiment 1, the subjects in the biased-immediately-informed and the biaseddelayed-informed conditions were significantly different, with those in the latter condition being more accurate on the final test. In Experiment 2, reminding subjects of the warning in the former condition eliminated this difference, which would seem to confirm our speculation that some of these subjects simply forgot about the warning in the first experiment.

As in Experiment 1, the proportions of correct and biased answers led to the same conclusions as the combination scores, although the correct and biased answers did not account for nearly all the responses, as had been the case in the earlier experiment. In addition, an analysis using all critical items (i.e., not restricting final test data to initially correct items) yielded a somewhat similar overall pattern of results, but the warning once again appeared to be less effective by this analysis. In fact, although the type of analysis made little difference in the case of the subjects in the immediate-warning condition, the subjects in the biased-delayed-informed condition were significantly less accurate than those in the neutral condition and were not superior to those in the biased condition when all responses were included. Thus, only the conditionalized analysis presents a clear indication that the warning was effective in overcoming the bias introduced in the narrative. Finally, the subjects' initial test scores did not differ significantly $(\mathrm{F}<2)$. The mean proportions correct were $.41, .41$, $.27, .44$, and .41 for the five conditions, in the order shown in Table 3.

Table 3

Mean Critical Item Scores for Initially Accurate Items in Experiment 2

\begin{tabular}{lcccrc}
\hline & \multicolumn{3}{c}{ Experimental Condition } \\
\cline { 2 - 6 } & $\begin{array}{c}\text { Neutral } \\
(\mathrm{N}=22)\end{array}$ & $\begin{array}{c}\text { Biased } \\
(\mathrm{N}=21)\end{array}$ & $\begin{array}{c}\text { Biased/Immediately } \\
\text { Informed } \\
(\mathrm{N}=21)\end{array}$ & $\begin{array}{c}\text { Biased/Delayed } \\
\text { Informed } \\
(\mathrm{N}=18)\end{array}$ & $\begin{array}{c}\text { Accurate/Delayed } \\
\text { Informed } \\
(\mathrm{N}=21)\end{array}$ \\
\hline Combination Score & 5.20 & 3.16 & 5.58 & 5.10 & 7.86 \\
Proportion Correct & .36 & .21 & .49 & .43 & .85 \\
Proportion Biased & .32 & .74 & .41 & .47 & .07 \\
\hline
\end{tabular}

Note-Combination scores were computed such that a 1 equals a high-confidence incorrect response and a 10 equals a high-confidence correct response. 
Table 4

Mean Narrative and Nonnarrative Item Scores for Initially Accurate Items in Experiment 2

\begin{tabular}{|c|c|c|c|c|c|}
\hline & \multicolumn{5}{|c|}{ Experimental Condition } \\
\hline & $\begin{array}{r}\text { Neutral } \\
(\mathrm{N}=22)\end{array}$ & $\begin{array}{c}\text { Biased } \\
(\mathrm{N}=21)\end{array}$ & $\begin{array}{c}\text { Biased/Immediately } \\
\text { Informed } \\
(\mathrm{N}=21) \\
\end{array}$ & $\begin{array}{c}\text { Biased/Delayed } \\
\text { Informed } \\
(\mathrm{N}=18)\end{array}$ & $\begin{array}{l}\text { Accurate/Delayed } \\
\text { Informed } \\
(\mathrm{N}=21)\end{array}$ \\
\hline & \multicolumn{5}{|c|}{ Narrative Items } \\
\hline Combination Score & 8.56 & 8.63 & 8.60 & 8.53 & 8.75 \\
\hline \multirow[t]{2}{*}{ Proportion Correct } & .90 & .86 & .90 & .87 & .91 \\
\hline & \multicolumn{5}{|c|}{ Nonnarrative Items } \\
\hline Combination Score & 7.41 & 7.98 & 7.67 & 8.21 & 8.29 \\
\hline Proportion Correct & .42 & .53 & .46 & .45 & .54 \\
\hline
\end{tabular}

Note-Combination scores were computed such that a 1 equals a high-confidence incorrect response and a 10 equals a high-confidence correct response.

Returning to the principal analysis, the subjects in the accurate-delayed-informed condition were found to be significantly more accurate than those in all other conditions. Although there was no accurate-noninformed condition to compare with directly, the extremely high accuracy suggests that the subjects did not reject the critical narrative information. The fact that these subjects did not reject the narrative descriptions of these details when they were accurate constitutes further evidence that the rejection of the narrative information is selective. Similar evidence against the narrative-rejection hypothesis was obtained from noncritical items. The five questions on both the initial and the final tests that ad. dressed accurately described information and the five questions about facts not mentioned in the narrative were examined. Combination scores and proportion correct scores were computed separately for these narrative and nonnarrative questions; only initially accurate items (note that initial and final versions of test questions were identical in this experiment) were used in the computation.

The means for these dependent measures are shown in Table 4. A two-way mixed analysis of variance was done on the combination scores, with condition as the between-subjects variable and type of question (narrative vs. nonnarrative) as the within-subjects variable. It yielded no significant main effect of condition $(F<1)$, a significant main effect of item type $[F(1,112)=$ $12.64, \mathrm{p}<.001]$, with subjects being more accurate on narrative items than on nonnarrative items ( $8.62 \mathrm{vs.}$ 7.92 , respectively), and no significant interaction between condition and item type $(F<1)$. The narrativerejection hypothesis would predict an interaction such that the three informed conditions would yield low accuracy on narrative items (due to the nonselective rejection of the accurate narrative information), but no differences across conditions for nonnarrative items. Clearly, this result was not obtained. The lone significant effect obtained in the above analysis was that of item type. This result has no bearing on the questions at hand, and it is not surprising that the subjects were more accurate on items that were reviewed for them in the narrative than on those items not reviewed.

\section{GENERAL DISCUSSION}

The subjects in our experiments were able to overcome the biasing effects of a misleading postevent narrative when they were given a warning within $45 \mathrm{~min}$ after having read the narrative. This finding was obtained in two separate experiments that used different events to be remembered and tested memory for objects, actions, and colors. The fact that warned subjects responded as accurately as subjects who had never been exposed to the biasing information implies that the original and narrative versions of the event must coexist in memory, and that subjects can retrieve these two representations and determine the source of each.

Why were we successful in demonstrating the existence of original memories, when previous attempts had failed? First, our warning clearly stated that some inaccuracies did exist in the narrative, making the effort at searching memory appear worthwhile to subjects, and at the same time implying that they should compare the two versions of the event. Previous warning studies (Greene et al., 1982; Loftus, 1979b) provided rather ambiguous warnings that might not have yielded such an effort, and other studies, for example, those using incentives to encourage retrieval of original memories (Loftus, 1979a), may not have directed the search efforts adequately. A second improvement over the previous studies involved the data analysis. Since one can only expect to retrieve from episodic memory that which has been encoded, tests for the retrieval of original memories should be done only on material for which a subject has demonstrated some initial memory. We therefore restricted our analyses to only those items on which each subject had been shown to be accurate shortly after the event. Admittedly, performance on this initial accuracy test is not a perfect measure of what each subject has stored in memory. But some measure, even an imprecise one, must be used to reduce the num- 
ber of items for which no retrieval technique could possibly work. The failure of previous studies to find successful retrieval of original memories may have been due to the inclusion of tests for memories that a particular subject could not possibly recover, since such memories never existed in the first place. Indeed, unrestricted analyses of the present experiments, using all critical items, yielded only equivocal support for successful warning effects, whereas the conditionalized analyses provided a clear-cut pattern that supported such effects. It should be acknowledged, however, that the additional rehearsal provided by the initial test (and successful retrieval) may have strengthened the original memories (Whitten \& Bjork, 1977). Thus, coexistence may be limited to well-encoded material.

There were, of course, other differences between the present study and the previous research. One difference of potential importance concerns the retention intervals. The interval between the event and the postevent information was 2 days in our experiments and 1 day or less in the previous experiments. It is unclear what effect, if any, this difference might have had. Perhaps the longer interval allows for greater differentiation of the two memory representations. If this is the case, then the conflicting results might be explained. But note that such an explanation does not deny the fact that original memories can be recovered; it merely points out that there may be temporal constraints on when this is possible. How postevent information differs in its impact at different points in time is an issue clearly in need of further study (see Dooling \& Christiaansen, 1977a, and Loftus et al., 1978).

Does the present evidence for coexistence of memories also argue that memories are permanent? Although our findings are consistent with the permanent-memory notion, one must keep in mind that there was never more than $45 \mathrm{~min}$ between the postevent narrative and the warning in our experiments. Thus, we have demonstrated that memory for the original event can coexist with the subsequent information up to $45 \mathrm{~min}$. In light of this time limit, our findings do not present very convincing evidence for the permanence of memories. Instead, we suggest a middle ground between the permanent existence of all memories, which is at least implied by the coexistence hypothesis, and the immediate and irreversible updating proposed by the alteration hypothesis. Perhaps when we update a memorial representation of an event, the original information is indeed rendered less accessible than the new information. Even though it is not erased from memory, the original information may be forgotten more rapidly than its replacement. We can speculate on one means by which this might occur. In terms of the current schema approaches to memory, updating memory would probably result in the new information's replacing the original in a particular slot of the event schema. From this perspective, the new information would be more accessible because it would be part of the organized schema, which can serve as a retrieval network. Beyond this accessibility difference, however, there also would likely be differences in forgetting. The beneficial effects on retention of integrating facts into a coherent whole are well documented (e.g., Bransford \& Johnson, 1972; Dooling \& Lachman, 1971), and therefore the original information, since it is no longer integrated into the schema, might be the more rapidly forgotten. The alternative position we have sketched might be called the "temporary-coexistence hypothesis." Although the present evidence does not allow one to choose between this temporary-coexistence hypothesis and the permanent-coexistence hypothesis, the former is at least as feasible and seems to be more compatible with other evidence that memory becomes more internally consistent and thematic over time (e.g., Bartlett, 1932; Christiaansen, 1980; Dooling \& Christiaansen, 1977b; Kintsch \& van Dijk, 1978; Spiro, 1980).

As the above discussion suggests, the time frame for effective memory editing is an important unresolved issue. But there are other important questions, as well. Can misleading details be edited from memory when they were encoded as presuppositions of questions instead of being part of a narrative description as in the present study? The latter might allow for better comparison with the event memory. What happens when multiple postevent sources influence memory? If warned that only one source was inaccurate, can one still edit the critical information from memory? Does the ability to edit extend to distortions in memory created in an indirect manner by the postevent information (Christiaansen et al., 1983; Loftus \& Palmer, 1974)? Obviously there is much work to be done to delineate the conditions under which memory editing is possible.

\section{REFERENCE NOTE}

1. Loftus, E. F. Personal communication, September 7, 1982.

\section{REFERENCES}

Bartletr, F. C. Remembering. Cambridge: Cambridge University Press, 1932.

Bransford, J. D., \& Johnson, M. K. Contextual prerequisites for understanding: Some investigations of comprehension and recall. Journal of Verbal Learning and Verbal Behavior, 1972, 11, 717-726.

Christianansen, R. E. Prose memory: Forgetting rates for memory codes. Journal of Experimental Psychology: Human Learning and Memory, 1980, 6, 611-619.

Christinansen, R. E., Sweeney, J. D., \& Ochalek, K. Influencing eyewitness descriptions. Law and Human Behavior, 1983, 7, 59-65.

Dodd, D. H., \& Bradshaw, J. M. Leading questions and memory: Pragmatic constraints. Journal of Verbal Learning and Verbal Behavior, 1980, 19, 695-704.

Dooling, D. J., \& Christiannegn, R. E. Episodic and semantic aspects of memory for prose. Journal of Experimental Psychology: Human Learning and Memory, 1977, 3, 428-436. (a)

Dooline, D. J., \& Christianisen, R. E. Levels of encoding and retention of prose. In G. H. Bower (Ed.), The psychology of learning and motivation (Vol. 11). New York: Academic Press, 1977. (b)

Doolina, D. J., \& Lachman, R. Effects of comprehension on 
retention of prose. Journal of Experimental Psychology, 1971, 88, 216-222.

Greene, E., FlynN, M. S., \& Loftus, E. F. Inducing resistance to misleading information. Journal of Verbal Learning and Verbal Behavior, 1982, 21, 207-219.

Kintsch, W., \& van DiJK, T. A. Toward a model of text comprehension and production. Psychological Review, 1978, 85, 363-394.

LofTUs, E. F. Leading questions and the eyewitness report. Cognitive Psychology, 1975, 7, 560-572.

Loftus, E. F. Eyewitness testimony. Cambridge, Mass: Harvard University Press, 1979. (a)

LofTus, E. F. Reactions to blatantly contradictory information. Memory \& Cognition, 1979, 7, 368-374. (b)

Loftus, E. F., \& Greene, E. Warning: Even memory for faces may be contagious. Law and Human Behavior, 1980, 4, 323-334.

Loftus, E. F., \& Loftus, G. R. On the permanence of stored information in the human brain. American Psychologist, 1980, $35,409-420$.

Loftus, E. F., Miller, D. G., \& BurN8, H. J. Semantic integration of verbal information into a visual memory. Journal of Experimental Psychology: Human Learning and Memory, 1978, 4, 19-31.

Loftus, E. F., \& Palmer, J. E. Reconstruction of automobile destruction: An example of the interaction between language and memory. Journal of Verbal Learning and Verbal Behavior, $1974,13,585-589$.
Powers, P. A., Andriks, J. L., \& Loftus, E. F. Eyewitness accounts of males and females. Journal of Applied Psychology, $1979,64,339-347$.

SpIRo, R. J. Accommodative reconstruction in prose recall. Journal of Verbal Learning and Verbal Behavior, 1980, 19, 84-95.

WhitTen, W. B., II, \& Buonk, R. A. Learning from tests: Effects of spacing. Journal of Verbal Learning and Verbal Behavior, $1977,16,465-478$.

\section{NOTES}

1. In the one study that did include an initial accuracy test, that test did not ask about the specific critical details (see Loftus, $1979 \mathrm{~b}$, p. 370) because it was thought that providing such a rehearsal might make it difficult to later mislead subjects about these facts (Loftus, Note 1). In part, it was our concern for this same potential problem that led us to employ a longer retention interval (from initial test to biasing narrative) than had been used in previous studies, thus reducing the effect of the rehearsal.

2. Copies of the narratives used in both experiments may be obtained from the first author.

3. The analyses done on the combination scores are reported in this paper, but analyses of proportions correct yielded essentially the same results in each case.

(Manuscript received October 12, 1982; revision accepted for publication May 13, 1983.) 